\title{
AUTOAVALIAÇÃO VOCAL E QUALIDADE DE VIDA EM VOZ DE INDIVÍDUOS HIPERTENSOS
}

\author{
Self-perception vocal and quality of life \\ in the hypertensive individuals
}

\author{
Vanessa Veis Ribeiro (1), Angelika Bissolotti dos Santos ${ }^{(2)}$, Tatiane Prestes ${ }^{(3)}$, \\ Eveline Bonki ${ }^{(4)}$, Luciana Carnevale ${ }^{(5)}$, Ana Paula Dassie Leite ${ }^{(6)}$
}

\begin{abstract}
RESUMO
Objetivo: obter dados de autoavaliação vocal e qualidade de vida em voz de indivíduos hipertensos Método: trata-se de estudo transversal, exploratório e descritivo. Participaram 84 indivíduos, 33 homens e 51 mulheres, de 54 a 87 anos (média de 68 anos) que foram divididos em: Grupo Pesquisa (GP) - 42 indivíduos portadores de hipertensão arterial e que recebiam tratamento medicamentoso específico para a doença há pelo menos um ano; Grupo Controle (GC): 42 indivíduos não hipertensos e com bom estado de saúde geral autorreferido. Todos os participantes responderam a um questionário composto por perguntas objetivas sobre autoavaliação vocal e ao protocolo Qualidade de Vida em Voz (QVV). Resultados: não foram observadas diferenças quanto à autoavaliação vocal de indivíduos do GP e GC $(p=0,075)$. Os escores médios totais obtidos no QVV também foram semelhantes entre os grupos $(p=0,080)$. Houve diferença entre os grupos quanto à autopercepção do sintoma de "voz fraca", que teve maior ocorrência no GP $(p=0,015)$. Conclusão: a utilização de medicamentos específicos para a hipertensão não promoveu diferenças na autoavaliação vocal e na qualidade de vida do grupo estudado, com exceção da percepção de "voz fraca". Indivíduos hipertensos e não hipertensos apresentaram escores abaixo do que se espera para sujeitos com vozes saudáveis e sem queixas vocais.
\end{abstract}

DESCRITORES: Disfonia; Distúrbios da Voz; Hipertensão; Qualidade da Voz; Voz

(1) Acadêmica do Curso de Fonoaudiologia da Universidade Estadual do Centro-Oeste - UNICENTRO - Irati - Paraná (PR), Brasil.

(2) Acadêmica do Curso de Fonoaudiologia da Universidade Estadual do Centro-Oeste - UNICENTRO - Irati - Paraná (PR), Brasil.

(3) Acadêmica do Curso de Fonoaudiologia da Universidade Estadual do Centro-Oeste - UNICENTRO - Irati - Paraná (PR), Brasil.

(4) Acadêmica do Curso de Fonoaudiologia da Universidade Estadual do Centro-Oeste - UNICENTRO - Irati - Paraná (PR), Brasil.

(5) Fonoaudióloga; Professora Colaboradora do Curso de Fonoaudiologia da Universidade Estadual do Centro-Oeste - UNICENTRO, Irati, PR, Brasil; Doutora em Linguística Aplicada e Estudos da Linguagem pela PUC-SP.

(6) Fonoaudióloga; Professora Assistente do Curso de Fonoaudiologia da Universidade Estadual do Centro-Oeste - UNICENTRO, Irati, PR, Brasil; Mestre em Saúde da Criança e do Adolescente pela UNICAMP, SP.

Conflito de interesses: inexistente

\section{INTRODUÇÃO}

A hipertensão arterial (HA) é uma doença crônica, assintomática (na maior parte dos casos) e de grande incidência na população ${ }^{1}$. Em todo o mundo, diversos estudos epidemiológicos vêm sendo desenvolvidos com o intuito de compreender os fatores de risco e as direções de tratamento ao indivíduo hipertenso ${ }^{2,3}$.

Pesquisas recentes acerca da prevalência de casos de hipertensão arterial, têm obtidos valores que variam entre 10 e $40 \%$ da população ${ }^{3,4}$ e estima-se que em 2025 estes números serão consideravelmente maiores ${ }^{5}$. Alguns dos fatores de risco para a hipertensão arterial são obesidade, diabetes e consumo abusivo de álcool ${ }^{2,3,6,7}$.

A hipertensão arterial pode interferir na qualidade de vida do indivíduo, pois aumenta as chances de ocorrência de infarto do miocárdio, derrame cerebral, doenças renais e visuais ${ }^{8}$. $O$ tratamento 
da hipertensão pode ser realizado de forma farmacológica e não farmacológica ${ }^{1}$. O tratamento com medidas não-farmacológicas inclui medidas como redução de peso, realização de exercícios físicos, redução do consumo de sódio e diminuição do stress na vida diária ${ }^{1,9}$. O tratamento com substâncias farmacológicas inclui principalmente o uso de drogas anti-hipertensivas, como bloqueadores dos receptores de angiotensina, betabloqueadores, bloqueadores de canais de cálcio e diuréticos ${ }^{10,11}$.

Como a HA é uma doença crônica, a utilização de fármacos é fundamental para controlá-la. Indivíduos hipertensos passam a utilizar medicamentos por toda a vida e, atualmente, já existem relatos sobre os efeitos colaterais dos anti-hipertensivos na qualidade de vida do indivíduo ${ }^{8}$. Dentre os diversos efeitos colaterais já descritos, são mencionadas as alterações de trato vocal ${ }^{12,13}$.

Os medicamentos diuréticos podem causar desidratação do trato vocal e, quando combinados com outros medicamentos hipertensivos, como as angiotensinas e os inibidores de enzima conversora (captopril e enalapril) podem gerar rouquidão, tosse e afonia ${ }^{14}$. Tais alterações podem interferir negativamente na função vocal do indivíduo.

Uma outra consequência da desidratação do trato vocal é a presença de xerostomia. Estudo internacional mostra que a hipofunção salivar pode aumentar o esforço durante a fonação e causar modificações em alguns parâmetros, como na extensão vocal referente a pitch e loudness ${ }^{15}$.

Alterações de voz podem influenciar negativamente na autoavaliação vocal. Atualmente, há protocolos bastante sensíveis que investigam o impacto de uma possível disfonia na vida do indivíduo, como o "Qualidade de Vida em Voz" (QVV) ${ }^{16}$. Estudo mostra que os índices de qualidade de vida em voz obtidos por indivíduos com vozes saudáveis ou sem queixas vocais são superiores aos índices obtidos por indivíduos disfônicos ${ }^{17}$. Assim, obter dados de autoavaliação de hipertensos pode contribuir para a reflexão acerca dos possíveis problemas de voz enfrentados por esse grupo.

Embora o presente estudo não tenha tido o objetivo de investigar os efeitos das drogas anti-hipertensivas, parte-se da idéia de que sua repercussão na função vocal (ressecamento do trato, maior esforço à fonação, modificações de parâmetros, etc.) poderia influenciar negativamente na autoavaliação dos portadores da doença. Desta forma, ter acesso a esses dados poderia contribuir para o delineamento de ações fonoaudiológicas junto ao grupo de hipertensos.

Diante do exposto, nosso objetivo foi obter dados referentes à autoavaliação vocal e qualidade de vida em voz de indivíduos hipertensos. Para tal caracterização, foram coletadas informações sobre sintomas, autopercepção sobre eventuais modificações vocais e restrições sociais (no que se refere à voz) referidas por essa população.

\section{MÉTODO}

Trata-se de estudo transversal, de caráter descritivo e exploratório. Participaram 84 indivíduos, 33 homens e 51 mulheres, de 54 a 87 anos (média 68 anos) que foram divididos em: Grupo Pesquisa (GP) - 42 indivíduos portadores de hipertensão arterial, média de idade de 69 anos, e que recebem tratamento medicamentoso específico para a doença há pelo menos um ano; Grupo Controle (GC): 42 indivíduos não hipertensos, com bom estado de saúde geral autorreferido e média de idade de 66 anos.

Foram adotados como critérios de exclusão para ambos os grupos: possuir patologia de origem neurológica, auditiva, neoplásica, ou qualquer outra doença que pudesse interferir significantemente no processo de comunicação ou na função vocal. Foram excluídos, ainda, os que possuíam hábito de tabagismo. Foram mantidos, em ambos os grupo, indivíduos com doenças comuns ao processo de envelhecimento (faixa etária de maior ocorrência de hipertensão), uma vez que controlar tais variáveis limitaria substancialmente o número de sujeitos da amostra. Para participar do GP, o indivíduo deveria fazer uso de medicamento hipertensivo (monoterapia ou combinação de medicamentos) há pelo menos um ano ininterruptamente.

A coleta foi realizada por conveniência, em locais públicos (praças, ruas, etc.). Todos os participantes responderam a um questionário composto por perguntas objetivas sobre autopercepção vocal (levantamento de sintomas e percepção em relação à qualidade da voz e eventuais modificações vocais ao longo do tempo). No total, 12 sintomas foram investigados: voz rouca, voz alterada, voz fraca, voz tem mudado, perda da voz, tosse, pigarro, sensação de boa seca, sensação de corpo estranho na garganta, saliva espessa, cansaço ao falar e dor ao falar. O participante deveria responder "sim" ou "não" para cada um dos sintomas questionados.

Além disso, todos os indivíduos responderam ao protocolo "Qualidade de Vida em Voz (QVV)", traduzido e validado para o português por Gasparini e Behlau ${ }^{16}$. O QVV é composto por 10 questões que investigam o impacto de um possível problema de voz na vida do sujeito. Foi realizado cálculo específico para estabelecimento dos escores. Foram obtidas as médias referentes aos domínios físico, sócio-emocional e total para o GP e o GC.

A pesquisa foi aprovada pelo Comitê de Ética em Pesquisa da Universidade Estadual do 
Centro-Oeste, sob número 075/2009. Todos os participantes assinaram o Termo de Consentimento Livre e Esclarecido (TCLE), de acordo com a Resolução 196/96 da Comissão Nacional de Ética em Pesquisa (CONEP).

Os dados coletados foram tabulados e as variáveis foram analisadas estatisticamente por meio de testes não paramétricos denominados "Teste de Mann-Whitney" e "Igualdade de Duas Proporções". Foi adotado nível de significância de $5 \%$, ou seja, todos os intervalos foram construídos com $95 \%$ de confiança estatística.

\section{RESULTADOS}

Quanto à faixa etária dos participantes, não houve diferença entre os grupos GP e GC $(0,099)$.
Também não houve diferença entre a proporção de idosos e não idosos nos dois grupos, sendo que seis indivíduos do GP e dez do GC tinham idade inferior a 60 anos $(p=0,266)$. No que se refere ao gênero, de maneira geral houve maior número de mulheres $(p=0,005)$. No entanto, não houve diferença $(p=0,118)$ quanto à proporção de homens e mulheres nos dois grupos (29 mulheres e 13 homens no GP; 22 mulheres e 20 homens no GP).

Todas as possibilidades de problemas de saúde e ingestão de outros medicamentos foram questionadas (Tabela 1). Observa-se que houve diferença entre os grupos apenas na referência de "problemas na coluna" e "problemas circulatórios". Quanto à ingestão de medicamentos, os grupos apresentaram-se de maneira semelhante, com baixa ocorrência em ambos.

Tabela 1 - Distribuição de dados referentes à saúde geral e medicamentos diários utilizados por hipertensos (GP) e não hipertensos (GC)

\begin{tabular}{|c|c|c|c|c|c|}
\hline \multirow{2}{*}{ Saúde Geral } & \multicolumn{2}{|c|}{ Hipertensos } & \multicolumn{2}{|c|}{ Não Hipertensos } & \multirow{2}{*}{ valor de $p$} \\
\hline & $\mathbf{n}$ & $\%$ & $\mathbf{n}$ & $\%$ & \\
\hline Rinite alérgica & 8 & $19,00 \%$ & 7 & $16,70 \%$ & 0,776 \\
\hline Artrite & 10 & $23,80 \%$ & 6 & $14,30 \%$ & 0,266 \\
\hline Distúrbios hormonais & 2 & $4,80 \%$ & 7 & $16,70 \%$ & 0,078 \\
\hline Diabetes & 3 & $7,10 \%$ & 5 & $11,90 \%$ & 0,457 \\
\hline Reumatismo & 6 & $14,30 \%$ & 6 & $14,30 \%$ & 1 \\
\hline Colesterol & 2 & $4,8 \%$ & 1 & $2,4 \%$ & 0,557 \\
\hline Problemas cardíacos & 10 & $23,80 \%$ & 10 & $23,80 \%$ & 1 \\
\hline Gastrite & 0 & $0,0 \%$ & 1 & $2,4 \%$ & 0,314 \\
\hline Gripes frequentes & 9 & $21,40 \%$ & 12 & $28,60 \%$ & 0,450 \\
\hline Depressão & 5 & $11,9 \%$ & 3 & $7,1 \%$ & 0,457 \\
\hline Problemas na bexiga & 0 & $0,0 \%$ & 1 & $2,4 \%$ & 0,314 \\
\hline Problemas circulatórios & 6 & $14,3 \%$ & 0 & $0,0 \%$ & $0,011^{*}$ \\
\hline Problemas de visão & 1 & $2,4 \%$ & 0 & $0,0 \%$ & 0,314 \\
\hline Asma & 1 & $2,4 \%$ & 3 & $7,1 \%$ & 0,306 \\
\hline Bronquite & 0 & $0,0 \%$ & 1 & $2,4 \%$ & 0,314 \\
\hline Problemas na coluna & 19 & $45,2 \%$ & 10 & $23,8 \%$ & $0,039^{*}$ \\
\hline \multirow{2}{*}{ Medicamentos diários } & \multicolumn{2}{|c|}{ Hipertensos } & \multicolumn{2}{|c|}{ Não Hipertensos } & \multirow{2}{*}{ valor de $p$} \\
\hline & $\mathbf{n}$ & $\%$ & $\mathbf{n}$ & $\%$ & \\
\hline Distúrbios hormonais & 1 & $2,40 \%$ & 2 & $4,80 \%$ & 0,557 \\
\hline Problemas cardíacos & 3 & $7,10 \%$ & 2 & $4,80 \%$ & 0,645 \\
\hline Diabetes & 2 & $4,8 \%$ & 3 & $7,1 \%$ & 0,645 \\
\hline Problemas na coluna & 0 & $0,00 \%$ & 1 & $2,40 \%$ & 0,314 \\
\hline Reumatismo & 1 & $2,4 \%$ & 0 & $0,0 \%$ & 0,314 \\
\hline Colesterol & 2 & $4,8 \%$ & 1 & $2,4 \%$ & 0,557 \\
\hline Depressão & 2 & $4,8 \%$ & 2 & $4,8 \%$ & 1,000 \\
\hline
\end{tabular}

* Valores estatisticamente significantes $(p \leq 0,05)$ - Teste de igualdade de duas proporções

Legenda : $\mathrm{n}=$ número de sujeitos 
Os resultados mostram que não existe diferença entre os grupos (GP e GC) quanto à quantidade média de sintomas e aos escores médios obtidos nos domínios físico, sócio-emocional e total do QVV
(Tabela 2). Do total de 12 sintomas questionados, obteve-se a mediana de 2 para o GP e 1,5 para o GC. O cálculo do valor de $p$ foi feito a partir da mediana devido ao alto desvio padrão obtido.

Tabela 2 - Comparação de grupos para quantidade de sintomas, QVV físico, QVV sócio-emocional e QVV total

\begin{tabular}{llccccc}
\hline & Grupos & Média & Mediana & $\begin{array}{c}\text { Desvio } \\
\text { Padrão }\end{array}$ & $\mathbf{n}$ & Valor de $\mathbf{p}$ \\
\hline \multirow{2}{*}{ Quantidade de sintomas } & GP & 2,79 & 2 & 2 & 42 & 0,075 \\
& GC & 2,18 & 1,5 & 1,94 & 42 & \\
QVV Físico & GP & 85,12 & 91,7 & 17,75 & 42 & 0,108 \\
\multirow{3}{*}{ QVV Sócio-Emocional } & GC & 88,79 & 97,9 & 18,16 & 42 & \\
& GP & 90,93 & 100 & 19,48 & 42 & 0,4 \\
QVV Total & GC & 90,48 & 100 & 20,66 & 42 & \\
& GP & 87,82 & 93,7 & 17,43 & 42 & 0,080 \\
\hline
\end{tabular}

* Valores estatisticamente significantes $(p \leq 0,05)$ - Teste Mann-Whitney

Legenda : QVV = qualidade de vida em voz; GP = grupo pesquisa; $\mathrm{GC}=$ grupo controle; $\mathrm{n}=$ número de sujeitos

Quanto à autopercepção vocal referente a sinais e sintomas laríngeos e vocais, observa-se que o sintoma de "voz fraca" foi mais referido pelos indivíduos do GP $(\mathrm{p}=0,015)$ (Tabela 3). Os outros sintomas apresentaram-se de maneira semelhante nos dois grupos.

Os dados mostram que a percepção de indivíduos hipertensos e não hipertensos quanto a possíveis alterações vocais apresentadas por eles e modificações que a voz vem tendo ao longo do tempo é bastante semelhante $(p=0,108$ e $p=0,381$, respectivamente).

É possível observar, ainda, que os sintomas mais referidos pelos indivíduos de ambos os grupos foi o de "boca seca" (GC e GP =13), "tosse" (GP=13 e $\mathrm{GC}=12$ ) e "pigarro" (GP e GC=12), não havendo diferença entre o GP e o GC.

Tabela 3 - Distribuição de média de sintomas referida pelos indivíduos do GP e GC

\begin{tabular}{lccccc}
\hline \multirow{2}{*}{ Tipo de sintoma } & \multicolumn{2}{c}{ Grupo Pesquisa } & \multicolumn{2}{c}{ Grupo Controle } & \multirow{2}{*}{ Valor de $\mathbf{p}$} \\
\cline { 2 - 5 } & $\mathbf{n}$ & $\%$ & $\mathbf{n}$ & $\%$ & 0,811 \\
\hline Tosse & 13 & 31,00 & 12 & 28,60 & 1 \\
Pigarro & 12 & 28,60 & 12 & 28,60 & 0,776 \\
Voz rouca & 8 & 19,00 & 7 & 16,70 & $0,015^{\star}$ \\
Voz fraca & 13 & 31,00 & 4 & 9,50 & 0,786 \\
Cansaço ao falar & 9 & 21,40 & 8 & 19,00 & 1 \\
Boca seca & 13 & 31,00 & 13 & 31,00 & 0,763 \\
Sensação de corpo estranho & 7 & 16,70 & 6 & 14,30 & 0,090 \\
Perda da voz & 5 & 11,90 & 1 & 2,40 & 0,763 \\
Saliva espessa & 7 & 16,70 & 6 & 14,30 & 0,078 \\
Dor ao falar & 7 & 16,70 & 2 & 4,80 & 0,381 \\
Voz tem mudado & 21 & 50,00 & 17 & 40,50 & 0,108 \\
Voz alterada & 18 & 42,90 & 11 & 26,20 & \\
\hline
\end{tabular}

*Valores estatisticamente significantes $(p \leq 0,05)$ - Teste Igualdade de Duas Proporções

Legenda : $\mathrm{n}=$ número de sujeitos 


\section{DISCUSSÃO}

Ainda é escasso o número de publicações acerca das características vocais e laríngeas de indivíduos hipertensos. Isso provavelmente se deve à dificuldade de controle das diversas variáveis que podem contribuir para a manifestação de um possível problema de voz nesses indivíduos. Portanto, com o presente estudo, tem-se o intuito de iniciar uma discussão a respeito dos distúrbios vocais e hipertensão, entendendo que este assunto ainda está longe de ser esgotado na literatura científica.

Há relatos na literatura de que a utilização de diversos tipos de medicamentos, dentre eles os anti-hipertensivos, pode contribuir para o desenvolvimento, manutenção ou intensificação de problemas vocais ${ }^{14,15}$. Como a maior parte dos indivíduos hipertensos é idosa, os indivíduos podem apresentar alterações morfológicas no trato vocal naturais do envelhecimento ${ }^{18}$ e os medicamentos poderiam contribuir para a manutenção do problema de voz. No entanto, no presente estudo esta hipótese não foi confirmada, já todos os cruzamentos, com exceção de um tipo de sintoma questionado (voz fraca), não houve diferença entre os indivíduos hipertensos e não hipertensos.

A média de sintomas vocais obtida pelos indivíduos dos dois grupos foi semelhante. É importante mencionar que um estudo com um maior número de sujeitos poderá contribuir para que se tenha mais clareza sobre esse resultado. No entanto, acredita-se que para o grupo de hipertensos pesquisado, os possíveis efeitos dos medicamentos e alterações vocais e laríngeas ainda não são significantes, ou seja, tais fatores ainda não têm desencadeado ou potencializado eventuais queixas tidas pelo grupo.

Considerando o total de 12 sintomas investigados, a média obtida pelo GP e GC (2,79 e 2,18, respectivamente) teve semelhanças e diferenças em relação às médias de estudos desenvolvidos com outras populações. Operadores de telesserviços, por exemplo, apresentam uma média de 2 sintomas $^{19}$ enquanto professores apresentam média de $3,7^{20}$ e cantores amadores de $7,78^{21}$.

No que se refere à qualidade de vida em voz, também foram obtidos escores totais semelhantes do QVV entre os indivíduos do GC e do GP. Estudo desenvolvido com outro grupo de indivíduos hipertensos concluiu que maioria deles refere impacto da disfonia em sua comunicação diária, apresentando índices reduzidos de qualidade de vida em voz ${ }^{22}$. É importante mencionar que os escores médios obtidos pelos indivíduos tanto do $\operatorname{GP}(87,82)$ quanto do $\operatorname{GC}(89,47)$ estão abaixo do que se espera para sujeitos com vozes saudáveis e sem queixas, que ocorre em torno de 95 pontos no QVV ${ }^{17}$. No entanto, indivíduos disfônicos tendem a apresentar médias ainda mais baixas, como mostram alguns estudos $^{23,24}$. Nesse sentido, não se pode inferir que o valor reduzido nos três domínios (físico, sócio-emocional e total) tenha relação direta com a hipertensão e torna-se possível a hipótese de que a faixa etária (semelhante nos dois grupos) tenha influenciado nos valores obtidos.

Nossos resultados apontam, ainda, que não houve diferença entre os grupos para a percepção quanto à qualidade vocal e quanto às mudanças vocais percebidas ao longo do tempo. Estudo com 103 idosas, que comparou análise perceptivo-auditiva e qualidade de vida em voz, concluiu que há predomínio de alteração vocal de grau leve a moderado $^{16}$. Desta maneira, justifica-se o fato de indivíduos do GC também terem apresentado percepção de alteração vocal e mudanças na voz. Ressalta-se que a maior parte dos estudos envolvendo características vocais de indivíduos idosos não fazem a exclusão de sujeitos com doenças crônicas como a hipertensão ${ }^{18,25,26}$.

Observa-se diferença entre os grupos para o sintoma de "voz fraca", com maior ocorrência no GP. É possível que devido à tentativa de compensar as modificações vocais que vêm sofrendo, tentando produzir uma voz melhor, os indivíduos desenvolvam ajustes inadequados que gerem fadiga vocal, cansaço e sensação de voz fraca. Além disso, estudos apontam redução da capacidade respiratória em idosos, com reduções do fluxo médio de ar, causando assim uma redução da intensidade da voz $^{15}$. No caso dos indivíduos do GP, acredita-se que a redução da capacidade vital, quando associada ao ressecamento do trato vocal causado pela utilização de medicamentos ${ }^{14,15}$, pode potencializar a percepção de alguns sintomas, como o de "voz fraca", por exemplo. No entanto, todos os outros sintomas foram referidos de forma semelhante entre os dois grupos, o que nos faz pensar que, de maneira geral, indivíduos hipertensos e não hipertensos não se diferenciam quanto à quantidade de sintomas referida.

Além da percepção sobre as alterações e as mudanças vocais, os sintomas mais referidos por indivíduos de ambos os grupos foram o de "boca seca" e "tosse". Assim, não é possível afirmar que a ingestão de medicamentos anti-hipertensivos, que diminuem assim a secreção salivar, potencializou a ocorrência destes sintomas, uma vez que no GP a manifestação foi semelhante ${ }^{15}$. Além disso, outros estudos já inferem que o sintoma "boca seca" também pode ser comum no grupo geral de idosos ${ }^{15}$.

Uma das opções a ser considerada em um próximo trabalho é tentar selecionar indivíduos 
hipertensos e não hipertensos não idosos, com idade inferior a 60 anos, o que pode contribuir para a redução dos fatores de confusão em relação aos sintomas e queixas apresentadas. Além disso, ter uma amostra maior e comparar os resultados de autoavaliação com a análise perceptivo-auditiva da voz desses indivíduos contribuirá para a fidedignidade das análises estatísticas.

\section{CONCLUSÃO}

A utilização de medicamentos específicos para a hipertensão não promoveu diferenças na autoavaliação vocal e na qualidade de vida do grupo estudado, com exceção da percepção de "voz fraca". Indivíduos hipertensos e não hipertensos apresentaram escores abaixo do que se espera para sujeitos com vozes saudáveis e sem queixas vocais. Isso pode ter ocorrido pela interferência de algumas variáveis não controladas no presente estudo, como a não restrição de determinadas faixas etárias, quaisquer outros problemas de saúde, sujeitos com dificuldades cognitivas e emocionais, etc. Por isso, sugere-se a realização de novos trabalhos, com maior controle de variáveis e realização de rastreios prévios, que tornem tanto o grupo pesquisa quanto o grupo controle mais homogêneos.

\begin{abstract}
Purpose: to get data of vocal self-assessment and voice quality of life in hypertensive individuals. Method: this is a cross-sectional, exploratory and descriptive study. There were 84 individuals ,33 men and 51 women, 54 to 87 years (68 years-old average) who were divided into: Study Group (SG) -42 individuals with hypertension and receiving specific drug treatment for this disease for at least one year; Control Group (CG): 42 non-hypertensive individuals with good self-reported general health. All participants answered a questionnaire with questions about vocal self-assessment and Voice-Related Quality of Life protocol (V-RQOL). Results: it was not observed differences in vocal self-assessment of individuals in groups $S G$ and $C G(p=0,075)$. Total average scores obtained in V-RQOL were also similar between both groups $(p=0,08)$. There was a difference between the groups regarding the self perception of the symptom of "a weak voice", which was more frequent in the $S G(p=0,015)$. Conclusion: the use of specific medications for hypertension didn't cause differences in vocal self-assessment and quality of life of the studied group, except for the perception of "weak voice". Non-hypertensive and hypertensive subjects had lower scores than subjects with healthy voice and without vocal complaints.
\end{abstract}

KEYWORDS: Dysphonia; Voice Disorders; Hypertension; Voice Quality; Voice

\section{REFERÊNCIAS}

1. Miranda RD, Perrotti TC, Bellinazzi VR, Nobrega TM, Criedoroglo MS, Toniolo Neto J. Hipertensão arterial no idoso: peculiaridades na fisiopatologia, no diagnóstico e no tratamento. Rev Bras Hipertens. 2002; 9 (3): 293-300.

2. Al Ghatrif M, Kuo YF, Al Snih S, Raji MA, Ray LA, Markides. KS Trends in hypertension prevalence, awareness, treatment and control in older Mexican Americans, 1993-2005. Ann Epidemiol. 2011;21(1):15-25.

3. Glasser SP; Judd S; Basile J; Lackland D; Halanych J; Cushman M; Prineas R; Howard V; Howard G Prehypertension, racial prevalence and its association with risk factors: Analysis of the
REasons for Geographic And Racial Differences in Stroke (REGARDS) study. Am J Hypertens. 2011;24(2):194-9.

4. Meng $X J$, Dong $G H$, Wang $D$, Liu $M M$, Lin $Q$, Tian S, Xu LX, Hou H, Ren YF, Lee YL. Prevalence, awareness, treatment, control, and risk factors associated with hypertension in urban adults from 33 communities of China: the CHPSNE study. J Hypertens. 2011;29(7):1303-10.

5. Twagirumukizaa $M$, Bacquerb DD, Kipsc JG, Backerb G, Stichelec RV and Van Bortelc LM. Current and projected prevalence of arterial hypertension in sub-Saharan Africa by sex, age and habitat: an estimate from population studies $\mathrm{J}$ Hypertens. 2011; 29:1243-52. 
6. Targa LV. A avaliação da pressão arterial por agentes comunitários pode ser uma estratégia útil para o cuidado da saúde. Rev. Bras. Med. Fam. e Com. 2006; 1(4):141-50.

7. Hartmann M, Dias-da-Costa JS, Olinto MTA, Pattussi MP, Tramontini A. Prevalência de hipertensão arterial sistêmica e fatores associados: um estudo de base populacional em mulheres no Sul do Brasil Cad. Saúde Pública. 2007; 23(8):1857-66. 8. Silva GPC, Caparroz-Assef SM, Bersani-Amado CA, Cuman RKN. Perfil e caracterização terapêutica dos pacientes internados no Hospital Universitário Regional de Maringá-PR. Arq. Ciênc. Saúde Unipar. 2002; 6(1):11-5.

9. Cavalcante MA, Bombig MTM, Luna filho B, Carvalho

ACC, de Paola AAV, Povoa R. Qualidade de vida de pacientes hipertensos em tratamento ambulatorial.

Arq Bras Cardiol. 2007; 89: 245-50.

10. Fajardo C. A importância da abordagem não farmacológica da hipertensão arterial na atenção primaria à saúde. Rev Bras Med Fam e Com. 2006;4(1):107-18.

11. Brennera R, Waeberb B, Allemanna Y. Medical treatment of hypertension in Switzerland - The 2009 Swiss Hypertension Survey (SWISSHYPE). Swiss Med Wkly. 2011;141:w13169.

12. Franco RJS. Crise hipertensiva: definição, epidemiologia e abordagem diagnóstica. Rev Bras Hipertens. 2002; 9(4): 340-5.

13. Braga NA, Pinho SMR, Tsuji DH, Sennes LU. Efeitos dos medicamentos na qualidade vocal e na laringe. In: Pinho, SMR. Tópicos em voz. Rio de Janeiro: Guanabara Koogan. 2001; s/n:18-27.

14. Abaza MM, Levy S, Hawkshaw MJ, Sataloff RT. Effects of Medications on the Voice. Otolaryngol Clin N Am. 2007; 40:1081-90.

15. Roh JL, Kim HS, Kim AY. The Effect of Acute Xerostomia on Vocal Function. Arch Otolaryngol Head Neck Surg. 2006;132:542-6.

16. Gasparini G, Behlau M. Quality of Life: Validation of the Brazilian Version of the Voice-Related Quality of Life (V-RQOL) Measure. Journal of Voice. 2009; 23:76-81.

17. Behlau M, Oliveira G, Santos LMA, Ricarte A. Validação no Brasil de protocolos de autoavaliação do impacto de uma disfonia. Pró-Fono. 2009; 21(4):326-32.

18. Soares EB, et. al. Hábitos vocais em dois grupos de idosos. Rev. CEFAC. 2007; 9(2):221-7.

19. Dassie-Leite AP, Lourenço L, Behlau M. Relação entre dados ocupacionais, sintomas e avaliação vocal de operadores de telesserviços. Rev. Soc. Bras. Fonoaudiol. 2011; 16(1): 59-63.

20. Behlau M, Zambon F, Guerrieri AC, Roy N, GVP. Panorama epidemiológico sobre a voz do professor no Brasil. Anais do $17^{\circ}$ Congresso Brasileiro de Fonoaudiologia. Salvador, 2009.

21. Ribeiro VV, Santos ACB, Bonki E, Prestes T, Dassie-Leite AP. Identificação de problemas vocais enfrentados por cantores de igreja. Rev CEFAC. 2011 [no prelo].

22. Mendes L, Dassie-Leite AP. Caracterização vocal de indivíduos hipertensos. Anais do $18^{\circ}$ Congresso Brasileiro de Fonoaudiologia. Curitiba, 2010.

23. Kasama ST, BRASOLOTTO AG. Vocal perception and quality of life Percepção vocal e qualidade de vida. Pró-Fono Rev Atual Científica. 2007; 19(1):19-28.

24. Putnoki DS, Hara F, Oliveira G, Behlau M. Qualidade de vida em voz: o impacto de uma disfonia de acordo com gênero, idade e uso vocal profissional. Rev. Soc. Bras. Fonoaudiol. 2010; 15(4): 485-90.

25. Costa $\mathrm{HO}$, Matias C. Impacto da voz na qualidade da vida da mulher idosa. Rev. Brasileira de otorrinolaringologia. 2005; 71(2):172-8.

26. Gama ACC, Alves CFT, Cerceau JSB, Teixeira LC. Correlação Entre Dados Perceptivoauditivos e Qualidade de Vida em Voz de Idosas. Pró-Fono Revista de Atualização Científica. 2009; 21(2):125-30.

http://dx.doi.org/10.1590/S1516-18462012005000074

RECEBIDO EM: 01/09/2011

ACEITO EM: 18/12/2011

Endereço para correspondência:

Vanessa Veis Ribeiro

Rua Souza Naves, ํㅡ227, Jardim Califórnia

Irati - PR

CEP: 84500-000

E-mail: vanessaribeirooo@ hotmail.com 